\title{
Resolution of multiple substrate binding sites in cytochrome P450 3A4: the stoichiometry of the enzyme-substrate complexes probed by FRET and Job's titrationt
}

\author{
Harshica Fernando, James R. Halpert, and Dmitri R. Davydov * \\ Department of Pharmacology and Toxicology, The University of Texas Medical Branch, Galveston, \\ 301 University Blvd., Texas 77555
}

\begin{abstract}
To explore the mechanism of homotropic cooperativity in human cytochrome P450 3A4 (CYP3A4) we studied the interactions of the enzyme with 1-pyrenebutanol (1-PB), 1- pyrenemethylamine (PMA), and bromocriptine by FRET from the substrate fluorophore to the heme, and by absorbance spectroscopy. These approaches combined with an innovative setup of titration-by-dilution and continous variation (Job's titration) experiments allowed us to probe the relationship between substrate binding and the subsequent spin transition caused by 1-PB or bromocriptine or the TypeII spectral changes caused by PMA. The 1-PB-induced spin shift in CYP3A4 reveals prominent homotropic cooperativity, which is characterized by a Hill coefficient of $1.8 \pm 0.3\left(S_{50}=8.0 \pm 1.1\right.$ $\mu \mathrm{M})$. In contrast, the interactions of CYP3A4 with bromocriptine or PMA reveal no cooperativity, exhibiting $K_{\mathrm{D}}$ values of $0.31 \pm 0.08 \mu \mathrm{M}$ and $6.7 \pm 1.9 \mu \mathrm{M}$, respectively. The binding of all three substrates monitored by FRET in titration-by-dilution experiments at an enzyme:substrate ratio of 1 reveals a simple bimolecular interaction with $K_{\mathrm{D}}$ values of $0.16 \pm 0.09,4.8 \pm 1.4$, and $0.18 \pm 0.09$ $\mu \mathrm{M}$ for 1-PB, PMA, and bromocriptine respectively. Correspondingly, the Job's titration experiments showed that the 1-PB-induced spin shift reflects the formation of a complex of the enzyme with two substrate molecules, while bromocriptine and PMA exhibit 1:1 binding stoichiometry. Combining the results of Job's titrations with the value of $K_{\mathrm{D}}$ obtained in our FRET experiments, we demonstrate that the interactions of CYP3A4 with 1-PB obey a sequential binding mechanism, where the spin transition is triggered by the binding of 1-PB to the low-affinity site, which becomes possible only upon saturation of the high-affinity site.
\end{abstract}

\section{Keywords}

substrate binding; cooperativity; conformers; spin equilibrium

\begin{abstract}
Recent studies of function and regulation of cytochromes P450 reveal increasing attention to the mechanisms and pharmacological significance of homo- and heterotropic cooperativity observed in various mammalian $\mathrm{P} 450$ species (1-8). The most prominent examples of these phenomena are observed with cytochrome P450 3A4 (CYP3A4), the most abundant P450 in human liver. CYP3A4 is responsible for the metabolism of a broad range of drug substrates, and studies of cooperativity of this enzyme are of vital importance to our efforts to elucidate the mechanisms of drug metabolism in humans (9). However, despite extensive studies, a general mechanism of cooperativity remains obscure.
\end{abstract}

\footnotetext{
${ }^{\dagger}$ This research was supported by NIH grants GM54995 (JRH), and Center grant ES06676 (JRH).

*Corresponding author: E-mail: d.davydov@UTMB.edu. Tel.: (409) 772-9658; Fax: (409) 772-9642.
} 
The prevailing hypothesis is that cytochromes $\mathrm{P} 450$ exhibiting cooperativity accommodate multiple substrate molecules in one large binding pocket (10-12). A loose fit of a single substrate molecule requires the binding of a second ligand for efficient binding and/or catalysis (11-13). However, the possibility that P450 cooperativity reflects a true case of allostery, involving an effector-induced conformational transition in the enzyme has also been discussed $(12,14-17)$. In this case an effector-binding site might be remote from the active site (12). The observation of a distal binding site for progesterone in CYP3A4 (18) in a recently solved Xray structure provides important support for this possibility.

The analysis of P450 cooperativity from steady-state kinetics of substrate oxidation does not provide any information on the connection between the binding of substrates and effectors and subsequent transitions in the enzyme. Therefore, many recent efforts in the studies of P450 cooperativity are increasingly focused on the substrate binding stage of the catalytic cycle $(16,17,19-22)$. In the case of Type-I substrates these interactions are known to modulate the spin equilibrium of the enzyme, which is thought to be an important determinant of its catalytic efficiency and coupling (23-28). Homotropic cooperativity in substrate binding is revealed in sigmoidal dependencies of the substrate-induced spin transitions on the substrate

concentration. However, in the case of multi-site binding, these transitions might be mediated by ligand-induced rearrangements in the enzyme and not reflect the binding event per se.

Moreover, the implied conformational heterogeneity of CYP3A4, where the enzyme pool is thought to be represented by several persistent conformers with different properties $(16,29$ 31 ), adds further complexity to the interpretation of substrate-induced spectral changes. Thus, unraveling the relationship between the binding of each substrate and/or effector molecule and the spin equilibrium of the heme iron is essential for understanding the mechanisms of P450 cooperativity $(19,20,22,32)$.

It is therefore important to implement direct approaches to monitor substrate binding, as in our recent use of fluorescence resonance energy transfer (FRET) with cytochrome P450eryF (P450 107A1) from Saccharopolyspora erythraea (22). This soluble bacterial enzyme exhibits homotropic $(22,33,34)$ and heterotropic (5) cooperativity with several substrates and lacks the complexity of membranous monooxygenase systems, thus providing a valuable reference point for the studies of eukaryotic cytochromes P450. In our experiments we used 1-pyrenebutanol (1-PB), a fluorescent substrate (22) that exhibits FRET to the heme of P450eryF upon formation of the enzyme-substrate complex. The results suggest that the enzyme possesses two binding sites with different affinities for 1-PB, and the binding of this substrate to the higher-affinity binding site does not modulate the spin equilibrium. The 1-PB-induced spin shift is thus observed only in the complex of P450 with two molecules of the substrate (22). This conclusion also appears to be valid for CYP3A4, as suggested by recent studies of the testosterone-induced spin shift utilizing a combination of absorbance and EPR spectroscopy (19), as well as interactions with testosterone and $\alpha$-naphthoflavone probed by the tryptophan fluorescence of the enzyme (3). A similar conclusion was also deduced by Baas and co-authors from studies of the testosterone-induced spin shift in monomeric CYP3A4 incorporated into a nanoscale lipid bilayer (Nanodisks) (20). The high value of the Hill coefficient obtained in this system $(n=2.24)$ prompted these authors to conclude that one molecule of CYP3A4 possesses at least three binding sites for testosterone. Based on this presumption, Baas and co-workers assessed the values of the individual dissociation constants from the fitting of the integral binding isotherm to a three-site model. However, none of the above studies provide direct knowledge of the parameters of individual binding events, which is essential for further elaboration of a mechanistic model of cooperativity in CYP3A4.

In the present study, we monitored enzyme-substrate interactions by FRET from the substrate fluorophore to the heme in combination with a novel design of titration experiments in order to resolve and characterize the individual binding events and to probe their relationship to the 
substrate-induced Type-I (spin shift) and Type-II spectral transitions using 1-pyrenebutanol (1-PB), 1-pyrenemethylamine (PMA), and bromocriptine as substrates. As in P450eryF, the binding of 1-PB to CYP3A4 results in displacement of the spin equilibrium towards the highspin state (Type I spectral transition) and reveals prominent homotropic cooperativity. Application of a titration-by-dilution approach (32) in FRET experiments with CYP3A4 allowed us to determine the dissociation constant $\left(K_{D 1}\right)$ of the stoichiometric 1:1 complexes of CYP3A4 with each substrate. Furthermore, we extended the titration-by-dilution approach to the spectrophotometric titration by introducing a specially designed optical cell with a vertical direction of the light beam, where the light path increases with the dilution of the sample. This novel design allowed us to employ a well-known principle of continuous variation (Job's titration) (35-37) to determine the stoichiometry of substrate binding to CYP3A4. We demonstrate that the substrate-induced spectral transitions in CYP3A4 reveal only one binding site for either PMA or bromocriptine, while the data obtained with 1-PB are consistent with a model of sequential binding to two binding sites, where the spin transition is observed upon the binding of 1-PB to the low-affinity site, which is triggered by the binding of the first substrate molecule at the high-affinity site.

\section{Experimental Procedures}

\section{Materials}

1-Pyrenebutanol (1-PB) and 1-pyrenemethylamine hydrochloride (PMA) were from Aldrich (New Jersey, USA), and bromocriptine mesylate was from Sigma chemicals (USA). All other chemicals used were of the highest grade available from commercial sources and were used without further purification.

\section{Expression and purification of CYP3A4}

The enzyme was expressed as the His-tagged protein in Escherichia coli TOPP3 and purified as described previously $(13,38)$. The protein was stored at $-80^{\circ} \mathrm{C}$ as $100-150 \mu \mathrm{M}$ solutions in $100 \mathrm{mM}$ HEPES buffer $(\mathrm{pH}=7.4)$, containing $10 \%$ glycerol $(\mathrm{v} / \mathrm{v}), 2 \mathrm{mM}$ TCEP (tris(2carboxy-ethyl) phosphine hydrochloride), and 1 mM EDTA (ethylenediaminetetraacetic acid).

\section{Experimental}

The absorbance and fluorescence spectra were measured with a MC2000-2 multi-channel CCD rapid scanning spectrometer (Ocean Optics, Inc., Dunedin, FL, USA) equipped with one absorbance and one fluorescence channel. We used an L7893 UV-VIS fiber-optics light source (Hamamatsu Photonics K. K., Hamamatsu City, Shizuoka, Japan) and a Model 63501 Pulsed Xenon Flashlamp Illuminator (Newport Corp., Stradford, CT) in absorbance and fluorescence experiments, respectively. The excitation of fluorescence was achieved using a Solar Blind 250-330 nm band-pass filter (cat. \#57860, Newport Corp., Stratford, CT). Alternatively, we used a combination of a computerized Hitachi F-2000 spectrofluorometer (Hitachi, Ltd., Tokyo, Japan) equipped with a custom-made thermostated cell holder and a magnetic stirrer in combination with an S2000 fiber optic CCD spectrometer (Ocean Optics, Dunedin, FL). The CCD spectrometer attached to a direct-path window of the cell holder was used to monitor the changes in the transmittance of the sample during the fluorometric titration. The excitation wavelength was set to $331 \mathrm{~nm}$ in the experiments with pyrenes and $320 \mathrm{~nm}$ in the experiments with bromocriptine. In both cases the excitation bandwidth was set to $10 \mathrm{~nm}$. The spectra of emission were recorded in the 360-570 nm range. Both MC2000-2 and Hitachi F-2000 designs used in our FRET experiments permitted simultaneous registration of the transmittance and the fluorescence emission spectra of the same sample, which allowed instant correction of the spectra of fluorescence for the changes in the intensity of the excitation light during the experiment. 
The spectrophotometric "titration-by-dilution" experiments were carried out with an S2000 spectrometer (Ocean Optics Inc.) equipped with a custom-designed fiber optic adapter for a 10-cm long cylindrical cell (Cell Type-521, NSG Precision Cells, Farmingdale, NY). In these experiments the light path of the sample increased simultaneously with dilution. We started these experiments by placing $300 \mu \mathrm{L}$ of enzyme-substrate mixture at the desired molar ratio into the cell. With our optical cell (internal diameter $8 \mathrm{~mm}$ ) this initial volume provides an optical path length of $0.6 \mathrm{~cm}$. The initial concentration of the enzyme was typically in the range of $7-15 \mu \mathrm{M}$ and was chosen to be higher than the expected value of $K_{\mathrm{D}}$, and to yield a maximal optical density in the Soret region of $\leq 1$ optical unit. Titration was made by gradual additions of aliquots of buffer until the cell was filled. As the cell has a total volume of $5.1 \mathrm{~mL}$, the final concentration of the enzyme/substrate mixture was 17-times lower than the initial one.

A similar technique was employed in our continuous variation (Job's titration) experiments. At the beginning of the experiment we placed into the cylindrical optical cell described above $0.5 \mathrm{~mL}$ of the enzyme solution at a concentration higher than the expected value of the dissociation constant. The titration was carried out by progressive addition of the solution of the substrate taken at the concentration equal to the initial concentration of the enzyme. The details of these designs will be described elsewhere.

All experiments were carried out at $25^{\circ} \mathrm{C}$ in $100 \mathrm{mM}$ HEPES buffer (pH 7.4), containing 1 $\mathrm{mM}$ DTT and $1 \mathrm{mM}$ EDTA (HEPES+ buffer). An $8-15 \mathrm{mM}$ stock solution of 1-PB in acetone was used in all experiments. The stock solution of PMA $(2-2.5 \mathrm{mM})$ was prepared in warm HEPES-buffer and kept warm during the experiments.

\section{Data Processing}

The series of absorbance and fluorescence spectra obtained in titration experiments were analyzed using principal component analysis method (PCA) as described previously $(39,40)$. This approach, which is illustrated in Supporting Information, allowed us to increase the signalto-noise ratio and improve the accuracy of the assay by sorting out the changes arising from the absorbance of the substrate and the changes in the light scattering during the experiment $(17,22)$. To interpret the changes in the spectra of absorbance in terms of the concentration of P450 low-spin, high-spin and P420 species we used a least-squares fitting of the spectra of the first and second principal components to the set of spectral standards of the pure high spin, substrate-free ferric low-spin $(16,39,40)$ and Type II-substrate-bound ferric low-spin CYP3A4. The latter spectral standard with the Soret band at $425 \mathrm{~nm}\left(\varepsilon=114 \mathrm{mM}^{-1} \mathrm{~cm}^{-1}\right), \alpha$-band at 571 $\mathrm{nm}\left(\varepsilon=10 \mathrm{mM}^{-1} \mathrm{~cm}^{-1}\right)$ and $\beta$-band at $538 \mathrm{~nm}\left(\varepsilon=15 \mathrm{mM}^{-1} \mathrm{~cm}^{-1}\right)$ was obtained from the titration of CYP3A4 with imidazole. The set of spectral standards of CYP3A4 used in this study is available in Supporting Information. The series of spectra obtained in dilution experiments with variable optical path length were normalized to the path length prior to further analysis. Application of PCA to the series of emission spectra obtained in FRET experiments resulted in the first principal component corresponding to the changes in substrate fluorescence and covering over $99 \%$ of the total spectral changes. The changes in the loading factor of the first principal component during the experiment were used to calculate the relative changes in the intensity of the fluorescence of substrate. All data treatment procedures and curve fitting were performed using our SPECTRALAB software package (39). The dependence of the fraction of the high spin heme protein or the loading factor of the first principal component on the substrate concentration was used to determine the parameters of the interactions from the fitting of these curves to an appropriate equation using a combination of Marquardt and NeldelMead non-linear least squares algorithms (39). 


\section{Results}

\section{Interactions of CYP3A4 with bromocriptine, 1-PB, and PMA monitored by absorbance spectroscopy}

In agreement with earlier findings (16), titration of CYP3A4 with bromocriptine revealed a profound displacement of the spin equilibrium of the heme iron towards the high-spin state (data not shown). Titration curves exhibit no cooperativity of the enzyme in these interactions and obey the equation for the isotherm of bimolecular association (41), p 73, eq. II-53):

$[E S]=\frac{[E]_{0}+[S]_{0}+K_{D}-\left\{\left([E]_{0}+[S]_{0}+K_{D}\right)^{2}-4 \cdot[E]_{0} \cdot[S]_{0}\right\}^{1 / 2}}{2}$

with $K_{D}=0.31 \pm 0.08 \mu \mathrm{M}$, as obtained by averaging the results of 9 individual experiments (Table 1).

As shown in Fig. 1a, changes in the absorbance spectra of CYP3A4 observed upon addition of 1-PB also exhibit a Type-I substrate binding, which is indicative of a substrate-induced displacement of the spin equilibrium of the enzyme. The substrate-induced increase in the fraction of the high-spin CYP3A4 is adequately approximated by the Hill equation (Fig. 1b). Averaging the results of 5 individual experiments gives an $S_{50}$ value and Hill coefficient $(n)$ of $8.0 \pm 1.1 \mu \mathrm{M}$ and $1.8 \pm 0.3$, respectively (Table 1). These parameters are close to those reported earlier for P450eryF ( $S_{50}$ and $n$ of $8.3 \mu \mathrm{M}$ and 2.4, respectively) (17).

The interaction of CYP3A4 with 1-pyrenemethylamine (PMA) is remarkably different from that observed with 1-PB. Binding of PMA results in Type-II spectral changes, which are indicative of the incorporation of a nitrogen atom of the substrate into the ligand sphere of the heme iron (Fig. 2a). This type of interaction is associated with a displacement of the Soret band of the low-spin heme protein from 418 to $426-427 \mathrm{~nm}$ (see Fig. 2a, inset). The titration curve in Fig. $2 b$ presents these changes in terms of a decrease in the fraction of the "normal" (waterligated) low-spin state of the enzyme and obeys the equation for the equilibrium of bimolecular association (Eq. 1) with a dissociation constant $\left(K_{D}\right)$ of $7.7 \pm 2.3 \mu \mathrm{M}$, as obtained by averaging the results of 3 individual experiments (Table 1).

\section{Interactions of CYP3A4 with 1-PB, PMA, and bromocriptine monitored by FRET to the heme of the enzyme}

As the interactions of pyrenes with CYP3A4 are expected to result in FRET from the substrate to the heme $(21,22,32)$, we monitored the changes in fluorescence of 1-PB and 1-PMA upon dilution of the enzyme-substrate mixtures. Thus, a series of emission spectra was taken at a constant molar ratio of enzyme:substrate and simultaneously decreasing concentrations of both compounds. In the case of multi-site binding this approach allows us to consider the binding events separately, provided that the affinities of the two sites remain considerably different irrespective of substrate saturation of the high-affinity site. When the enzyme and the substrate are mixed at a 1:1 molar ratio, the method is selective for the formation of a binary complex with substrate bound at the higher affinity site.

Changes in the specific fluorescence of 1-PB upon dilution of a 1:1 mixture with CYP3A4 are shown in Fig. 3a. The signal was normalized to the protein concentration and corrected for the internal filter effect as described in Experimental Procedures, so that in the absence of FRET the spectra are not expected to be affected by the concentration of the mixture. As shown in the inset in Fig. 3a, the principal component analysis applied to these series of spectra yields the first principal component signifying a concomitant decrease of all emission bands of 1-PB upon its interaction with CYP3A4. As shown in the inset in Fig. 3a, the second principal component deduced from this analysis may reveal some minor changes in the degree of 
excimerization of 1-PB during the experiment. However, the amplitude of this component was negligible, and it was not considered in further analysis. Although the decrease in the intensity of fluorescence of 1-PB observed here may result from several different reasons, FRET appears to be the most plausible cause. The principal component analysis did not reveal any significant changes in the shape of the emission spectra (Fig.3a and 3b, insets), which would be anticipated if the changes in the intensity of fluorescence were caused by a change in the environment of the fluorophore or formation of excimers.

The dependence of the relative intensity of 1-PB fluorescence on the concentration of enzymesubstrate mixture obeys the equation for the isotherm of binary association (Eq. 1). The value of the dissociation constant obtained by averaging the results of 3 individual experiments is equal to $0.16 \pm 0.09 \mu \mathrm{M}$. This value is lower than the $S_{50}$ determined by spectrophotometric titration by more than an order of magnitude (Table 1), thus suggesting a multi-site binding mechanism. We may conclude, therefore, that the binding of 1-PB to the high-affinity binding site detected in our FRET experiments does not result in the displacement of the spin equilibrium of the enzyme, similar to P450eryF $(17,22)$.

Similar to 1-PB, the changes in fluorescence observed upon the interaction of PMA with CYP3A4 are consistent with FRET from the substrate to the heme (Fig. 3b). Although the shape of the spectrum of the second principal component obtained in these experiment (Fig $3 \mathrm{~b}$, inset, dashed line) suggests some minor changes in the degree of excimerization of PMA, these changes were negligible. The changes in fluorescence of PMA observed in these experiments also obey the binary association isotherm (Eq. 1) with a dissociation constant of $4.8 \pm 1.4 \mu \mathrm{M}$, which is very close to the value found in our spectrophotometric titration experiments above. Thus, in contrast to 1-PB, the PMA binding event detected in FRET experiments is identical to the one causing the Type-II spectral transition in the enzyme.

Bromocriptine is also known as a fluorophore characterized with an excitation maximum around $320 \mathrm{~nm}$ and a broad emission maximum centered at $424 \mathrm{~nm}$, which considerably overlaps with the Soret absorbance bands of low- and high-spin CYP3A4. As shown in Fig. $3 \mathrm{c}$ the interaction of bromocriptine with CYP3A4 results in a considerable decrease in the intensity of fluorescence of the substrate, which is consistent with FRET to the heme of the enzyme. Titration curves obtained in a 1:1 dilution setup obey Eq. 1 with a dissociation constant of $0.18 \pm 0.09 \mu \mathrm{M}$, as obtained by averaging the results of 3 individual experiments. This value is consistent with the estimate obtained in our spectrophotometric titration (Table 1). Thus, similar to binding of PMA to CYP3A4, the enzyme interaction with bromocriptine monitored by FRET reflects the same substrate binding event as the bromocriptine-induced spin shift. Thus, our data obtained in FRET experiments are consistent with a model where the substrateinduced spectral transition in CYP3A4 reflects the formation of a binary complex with one molecule of either bromocriptine or PMA, while the 1-PB-induced spin-shift requires the saturation of two distinct substrate binding sites, whose affinities for this substrate are considerably different.

\section{Probing the substrate interactions with the low-affinity binding site by absorbance spectroscopy dilution experiments}

The dilution strategy used in our FRET experiments may also be used to probe the individual binding events by absorbance spectroscopy. In this case, however, we encounter an important difficulty arising from the decrease in the sample absorbance upon dilution. Within the range of enzyme concentrations used, the absorbance of the Soret band of P450 varies from $\sim 0.01$ to $\sim 2$ optical units. To overcome the experimental difficulties caused by such large variation of the spectral amplitude, we designed our experiments such that the optical path length increases together with the dilution of the sample. This was done with the use of an open 
cylindrical cell with a vertical direction of the light beam (from the surface to the bottom). In this case the amplitude of the spectra remains unchanged.

Our attempts to apply this approach to CYP3A4 interactions with 1-PB or PMA in 1:1 enzymesubstrate mixtures showed that the amplitude of the spectral transition observed is too low to be detected reproducibly (data not shown). However, the dilution setup described above was found applicable at excess substrate. While the studies of enzyme-substrate interactions by dilution of a 1:1 enzyme-substrate mixture are specific for determination of the $K_{\mathrm{D}}$ at the first (higher affinity) binding site, provided that the affinities of the two sites are considerably different, dilution experiments at excess substrate can be used to study substrate binding at a lower affinity site when the higher-affinity site is already saturated (32). For instance, assuming the dissociation constant for the first binding site to be equal to $0.2 \mu \mathrm{M}$, we may calculate with equation 1 that at a 10-fold molar excess of substrate over enzyme, $95 \%$ saturation of the higheraffinity site will be observed at an enzyme concentration of $0.42 \mu \mathrm{M}$. Accordingly, at higher enzyme concentrations the high-affinity binding site may be considered completely saturated, and the titration-by-dilution results will reflect the binding at the second (lower affinity) site only. Certainly, the resolution of two binding sites by this approach is possible only if the affinity of the second site for the substrate remains considerably lower that the one of the first site, even when the first site is occupied.

Dilution of 1:10 CYP3A4:1-PB or CYP3A4:PMA mixtures yielded spectral transitions similar to those observed in our titration experiments. Dilution of the CYP3A4:1-PB mixture resulted in decrease in the high-spin-specific absorbance bands, concomitant with an increase in the amplitude of those of the low-spin heme protein (Fig. 4a), while the dilution of the CYP3A4:PMA mixture was accompanied by changes indicative of the dissociation of the Type-II substrate complex (Fig 5a). In both cases the titration curves obey the equation of binary binding isotherm (Eq. 1) with dissociation constants of $6.1 \pm 1.7 \mu \mathrm{M}$ (1-PB) and $5.8 \pm$ $1.8 \mu \mathrm{M}$ (PMA) (Fig 4b, Fig 5b). Thus, the dissociation constant found in our FRET experiments with a $1: 1 \mathrm{CYP} 3 \mathrm{~A} 4: 1-\mathrm{PB}$ mixture $(0.16 \pm 0.08 \mu \mathrm{M})$ is considerably lower than the value obtained from absorbance measurements at excess substrate. Therefore, we may conclude that the 1-PB-induced Type-I spectral transition in CYP3A4 requires the binding of two molecules of the substrate, and the high-affinity interaction detected in our FRET experiments does not cause the modulation of the spin state of the enzyme by itself. In contrast, the estimates of the dissociation constant of CYP3A4 with PMA found in the dilution experiments with both 1:1 and 1:10 enzyme-substrate mixtures are similar, showing that the PMA-induced Type-II spectral transition in CYP3A4 reflects the formation of its binary complex with only one substrate molecule.

\section{Probing the stoichiometry of CYP3A4 interactions with substrates with the continuous variation (Job's titration) approach}

One of the approaches commonly used to determine the stoichiometry of enzyme-substrate employs the Job's method of continuous variation $(35,36)$ based on mixing the reactants in such a way that their molar ratio varies, but the total molar concentration remains constant. The position of the maximum of the bell-shaped curve of the dependence of the concentration of the product (enzyme-substrate complex in our case) on the molar ratio of the reactants reflects the stoichiometry of the interactions. Applying the technique proposed by Facchiano and Ragone for the studies of protein-protein interactions (37), we implemented Job's titration by means of the gradual mixing of enzyme and substrate solutions of similar concentration. To make this method applicable to absorbance spectroscopy, we combined it with the variable optical path length technique, as described in Experimental Procedures. We used this method to study the interactions of CYP3A4 with bromocriptine, 1-pyrenebutanol (1-PB), and 1pyrenemethylamine (PMA). The titration curves showing the changes in the concentration of 
the high-spin P450 state (for bromocriptine and 1-PB) or the concentration of the nitrogenligated low-spin heme protein (for PMA) are shown in Fig.6. In the case of bromocriptine and PMA, Job's titration gives bell-shaped curves with the maximum observed at a 1:1 enzyme:substrate ratio. The curve obtained with bromocriptine may be adequately fitted by the equation for the equilibrium of bimolecular association with a dissociation constant of 0.38 $\pm 0.03 \mu \mathrm{M}$. In the case of PMA, the fitting of yields a $K_{D}$ of $11.0 \pm 0.7 \mu \mathrm{M}$, although the asymmetric shape of the titration curve results in systematic deviation of the experimental points from the fitting curve. Thus, consistent with our conclusions above, the spectral changes observed with bromocriptine and PMA reflects the enzyme-substrate interactions in 1:1 stoichiometry. In contrast, in the case of 1-PB, the maximum of the Job's titration curve was found at approximately 2 -fold molar excess of the substrate over the enzyme, suggesting that the 1-PB-induced spin shift requires the formation of the enzyme substrate complex in 1:2 stoichiometry.

\section{Discussion}

The current concepts of the mechanisms of homotropic cooperativity in cytochromes $\mathrm{P} 450$ are based on the hypothesis of multiple substrate binding sites in one molecule of the heme protein $(12,20,42-47)$. However, to date there have been no reports on direct determination of the stoichiometry of P450 interactions with substrates or characterization of the individual binding sites in terms of their affinity for substrates. In this study we employed a combination of FRET and advanced UV-VIS spectroscopic titration techniques to elucidate the interactions of CYP3A4 with three different fluorescent substrates, of which only 1-PB was shown to possess detectable homotropic cooperativity.

Our results show that FRET from the substrate fluorophore to the heme of P450 may be successfully used to monitor the interactions with all three substrates. Furthermore, the use of a "titration-by-dilution" approach at a 1:1 enzyme-to-substrate ratio ensures that our FRET experiments are specific for the formation of a bimolecular enzyme-substrate complex. Therefore, our finding that the $K_{D}$ values of complexes with bromocriptine and PMA determined by FRET are similar to those determined from the spectrophotometric titrations with these substrates suggests that the spin shift and Type-II transition induced by bromocriptine and PMA, respectively, reflects directly the formation of the complex of CYP3A4 with one substrate molecule. In contrast, the fact that the FRET-derived dissociation constant of the complex with 1-PB is considerably lower than the $S_{50}$ value from the spin shift is consistent with a multi-site binding model. Furthermore the results suggest that the bimolecular interaction detected by FRET does not cause any displacement of the spin equilibrium by itself, being rather a prerequisite of the spin transition observed upon binding of the second 1-PB molecule.

Importantly, results of our determination of the stoichiometry of CYP3A4 interactions with 1PB, PMA, and bromocriptine are completely consistent with the above conclusions. The fact that the Job's titration with bromocriptine yields a symmetric bell-shaped curve suggests that the displacement of the spin state by this substrate results from a formation of the simple bimolecular enzyme-substrate complex. Moreover, the $K_{D}$ deduced from Job's titration with bromocriptine $(0.38 \pm 0.03 \mu \mathrm{M})$ is consistent with that determined from our spectrophotometric titration $(0.31 \pm 0.08 \mu \mathrm{M})$ and FRET dilution $(0.18 \pm 0.09 \mu \mathrm{M})$ experiments, which confirms the applicability of this simple bimolecular mechanism in this case. Although the stoichiometry of PMA interactions with CYP3A4 resulting in a Type-II spectral transition is also 1:1, the asymmetry of the Job's titration curve in this case suggests that the mechanism of interactions is more complex. There may be spectrally silent interactions of PMA at a second binding site having very low affinity for the substrate, or interactions with two different stable conformers in the enzyme (16). 
In contrast, the maximum at two-fold molar excess of 1-PB over the enzyme in the curve of Job's titration (Fig. 6c) clearly indicates that the 1-PB-induced spin transition requires the binding of two substrate molecules to one molecule of CYP3A4. The interactions of substrate with two binding sites in cytochrome P450 and subsequent spin transitions can be represented schematically as follows (32):

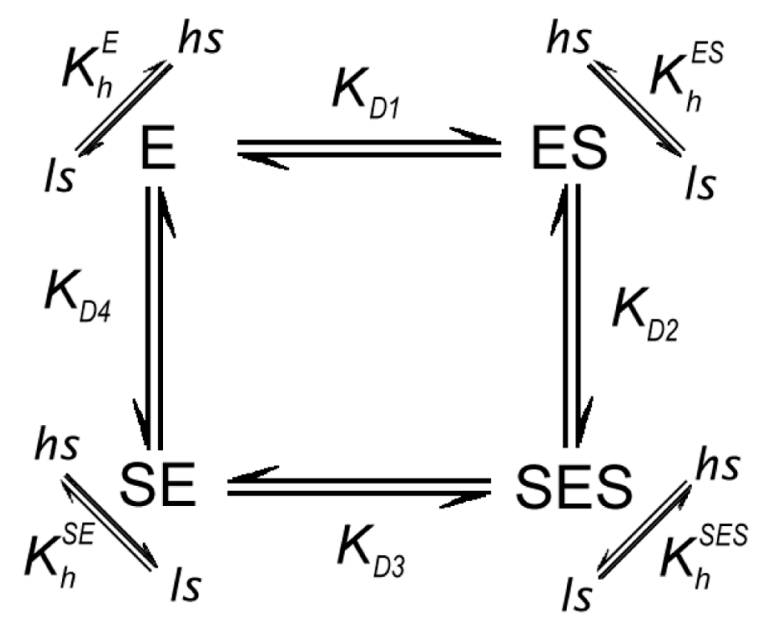

Here $E$ stands for the substrate-free enzyme; the complexes of the substrate bound at each of the two sites are designated by $E S$ and $S E ; S E S$ stands for the ternary complex with both binding sites occupied. $K_{\mathrm{D} 1}, K_{\mathrm{D} 2}, K_{\mathrm{D} 3}$ and $K_{\mathrm{D} 4}$ are the dissociation constants. The diagonal arrows show spin transitions, and $l s$ and $h s$ correspond to the low- and high-spin P450 respectively. $K_{h}^{E}, K_{h}^{E S}, K_{h}^{S E}$ and $K_{h}^{S E S}$ are the constants of spin equilibrium. In our studies of substrate interactions with P450eryF $(17,32)$ we suggested two extreme simplifications of this model termed the parallel and sequential binding mechanisms. The parallel binding model suggests independent binding of two substrate molecules at two binding sites where the substrate binding at each site has no effect on the interactions at the second one, so that $K_{\mathrm{D} 1}=K_{\mathrm{D} 3}, K_{\mathrm{D} 2}=$ $K_{\mathrm{D} 4}$. According to this model, which was analyzed in our early study on 1-PB interactions with P450eryF (22), the cooperativity of substrate-induced spin transitions is caused solely by the fact that the displacement of the spin equilibrium is observed in the ternary complex only. This model results in the following relationship between the total concentrations of substrate and the enzyme $\left([S]_{0}\right.$ and $[E]_{0}$ respectively) and the steady state concentration of the ternary complex ([SES]):

$$
\begin{gathered}
{[S]^{3}+\left(2 \cdot[E]_{0}-[S]_{0}+K_{\Sigma}\right)[S]^{2}+\left(K_{\Sigma}[E]_{0}-[S]_{o} K_{\Sigma}+K_{\Pi}\right)[S]-K_{\Pi}[S]_{o}=0} \\
\text { where } \mathrm{K}_{\Pi}=K_{D 1} \cdot K_{D 2} ; \quad K_{\Sigma}=K_{D 1}+K_{D 2}
\end{gathered}
$$

Another extreme case of a two-binding site model is sequential binding, where the binding at the second binding site is impossible without saturation of the higher-affinity effector site. This model suggests that $K_{\mathrm{D} 4}>>K_{\mathrm{D} 1}$ and $K_{\mathrm{D} 3}>>K_{\mathrm{D} 2}$, so that the pathways leading to the formation of SE may be neglected. To simplify the analysis further we proceeded with the assumption that the modulation of the spin equilibrium is observed in the ternary complex only and $K_{h}^{E}=K_{h}^{E S}$. Analyzing the scheme (2) with the above simplifications we obtained the following relationship between the steady state concentration of the ternary complex, that of the complex $E S([E S])$, and the total concentration of the enzyme and substrate (32):

$$
\begin{gathered}
2 \cdot[S E S]^{2}-\left([E]_{0}-[S E S]+K_{D 1}\right) \cdot[E S]-\left([S]_{0}+2 \cdot[E]_{0}+K_{D 2}\right) \cdot[S E S]+[S]_{0} \cdot[E]_{0}=0 \\
\text { where }[E S]=\frac{[S]_{0}}{2}-[S E S]-\left([S E S]^{2}-\left([S]_{0}+K_{D 2}\right) \cdot[S E S]+\frac{[S]_{0}^{2}}{4}\right)^{1 / 2}
\end{gathered}
$$


Although analytical solution of this equation for [SES] is intricate, it can be easily done by a numerical means (32).

To determine which of these two simplified models represents a closer match to the mechanism of CYP3A4 interactions with 1-PB we attempted to fit the results of our Job's titration experiments with 1-PB with both equation (3) and equation (4). As shown in Fig. 6c the sequential binding model provides better approximation of the experimental results than the parallel binding scheme. Moreover, the value of the low-affinity binding constant $\left(K_{D 2}\right)$ deduced from the fitting by the sequential binding model $(4.0 \pm 0.6 \mu \mathrm{M})$ is consistent with our estimate of $6.1 \pm 1.7 \mu \mathrm{M}$ deduced from the titration-by-dilution experiments, while the parallel binding model yields the value of $0.55 \pm 0.35 \mu \mathrm{M}$, which is much lower than observed. Therefore, we may infer that the interactions of 1-PB with CYP3A4 are consistent with the sequential binding of the substrate to two binding sites, which have considerably different affinities for the substrate. Thus, the mechanisms of interactions of 1-PB with CYP3A4 appear to be very close to those shown earlier for the interactions with P450eryF, where we also inferred the sequential binding mechanism by a somewhat different experimental approach $(22,32)$.

In conclusion, using a combination of FRET and novel titration approaches we were able to determine the stoichiometry of 1-PB interactions with CYP3A4 and demonstrate that the binding of this substrate is consistent with a sequential allosteric mechanism, where the binding at the second (low-affinity) binding site becomes possible (or largely facilitated) upon the saturation of the first (high-affinity) site. This conclusion is consistent with the recent analysis of the CYP3A4 interactions with testosterone by EPR and absorbance spectroscopy by Roberts et al. (19). Independently, the high value of the Hill coefficient obtained in the analysis of the interactions of testosterone with monomeric CYP3A4 incorporated into Nanodiscs lead Baas and co-workers to conclude that one molecule of the enzyme is able to bind three testosterone molecules (20). This inconsistency may be caused by a difference in the mechanisms of interactions of 1-PB as opposed to testosterone with CYP3A4, or, more likely, a difference between the monomeric state of CYP3A4 used in the work of Baas and co-authors and the oligomeric state of the enzyme used in our study. Observation of a distal binding site for progesterone in CYP3A4 by X-ray crystallography (18), suggests that the enzyme possess an additional (third) substrate binding site remote from the main substrate binding pocket. Oligomerization of the enzyme may result in blockage of this site in at least some of the subunits. Therefore, there may be an important difference between the monomers and oligomers of the enzyme in the mechanisms of cooperativity of substrate binding. This hypothesis is supported by our recent studies of the kinetics of dithionite-dependent reduction of oligomeric and Nanodisc-incorporated CYP3A4 (48). The question may be explored further by applying Job's titration technique to determine the stoichiometry of the interactions of substrates with monomeric CYP3A4 incorporated into Nanodiscs.

\section{Supplementary Material}

Refer to Web version on PubMed Central for supplementary material.

\section{ABBREVIATIONS AND TEXTUAL FOOTNOTES}

CYP3A4, cytochrome P450 3A4; Hepes, N-[2-Hydroxyethylpiperazine-N'-[2-ethanesulfonic acid]; DTT, dithiothreitol; EDTA, ethylenediaminetetraacetic acid.

\section{Acknowledgements}

The authors thank Dr. Wataru Honma and Ms. Nadezhda Davydova for their assistance in purification of the protein and FRET titration experiments. 


\section{References}

1. Atkins WM. Implications of the allosteric kinetics of cytochrome P450s. Drug Discovery Today 2004;9:478-484. [PubMed: 15149623]

2. Shou MG. The impact of cytochrome $\mathrm{P} 450$ allosterism on pharmacokinetics and drug-drug interactions. Drug Discovery Today 2004;9:636-637. [PubMed: 15279845]

3. Yoon MY, Campbell AP, Atkins WM. "Allosterism" in the elementary steps of the cytochrome P450 reaction cycle. Drug Metab. Rev 2004;36:219-230. [PubMed: 15237852]

4. Ekins S, Stresser DM, Williams JA. In vitro and pharmacophore insights into CYP3A enzymes. Trends Pharm. Sci 2003;24:161-166. [PubMed: 12707001]

5. Khan KK, Liu H, Halpert JR. Homotropic versus heterotopic cooperativity of cytochrome P450eryF: A substrate oxidation and spectral titration study. Drug Metab. Disp 2003;31:356-359.

6. Hutzler JM, Wienkers LC, Wahlstrom JL, Carlson TJ, Tracy TS. Activation of cytochrome P4502C9mediated metabolism: mechanistic evidence in support of kinetic observations. Arch. Biochem. Biophys 2003;410:16-24. [PubMed: 12559973]

7. Hutzler JM, Hauer MJ, Tracy TS. Dapsone activation of CYP2C9-mediated metabolism: Evidence for activation of multiple substrates and a two-site model. Drug Metab. Disp 2001;29:1029-1034.

8. Miller GP, Guengerich FP. Binding and oxidation of alkyl 4-nitrophenyl ethers by rabbit cytochrome P450 1A2: Evidence for two binding sites. Biochemistry 2001;40:7262-7272. [PubMed: 11401574]

9. Guengerich FP. Cytochrome P-450 3A4: regulation and role in drug metabolism. Annu. Rev. Pharmacol. Toxicol 1999;39:1-17. [PubMed: 10331074]

10. Shou M, Grogan J, Mancewicz JA, Krausz KW, Gonzalez FJ, Gelboin HV, Korzekwa KR. Activation of CYP3A4 - evidence for the simultaneous binding of 2 substrates. Biochemistry 1994;33:64506455. [PubMed: 8204577]

11. Korzekwa KR, Krishnamachary N, Shou M, Ogai A, Parise RA, Rettie AE, Gonzalez FJ, Tracy TS. Evaluation of atypical cytochrome P450 kinetics with two-substrate models: evidence that multiple substrates can simultaneously bind to cytochrome P450 active sites. Biochemistry 1998;37:41374147. [PubMed: 9521735]

12. Ueng YF, Kuwabara T, Chun YJ, Guengerich FP. Cooperativity in oxidations catalyzed by cytochrome P450 3A4. Biochemistry 1997;36:370-381. [PubMed: 9003190]

13. Harlow GR, Halpert JR. Analysis of human cytochrome P450 3A4 cooperativity: construction and characterization of a site-directed mutant that displays hyperbolic steroid hydroxylation kinetics. Proc. Natl. Acad. Sci. USA 1998;95:6636-6641. [PubMed: 9618464]

14. Schrag ML, Wienkers LC. Topological alteration of the CYP3A4 active site by the divalent cation Mg2+ Drug Metab. Disp 2000;28:1198-1201.

15. Atkins WM, Wang RW, Lu AYH. Allosteric behavior in cytochrome P450-dependent in vitro drugdrug interactions: A prospective based on conformational dynamics. Chem. Res. Toxicol 2001;14:338-347. [PubMed: 11304120]

16. Davydov DR, Halpert JR, Renaud JP, Hui Bon Hoa G. Conformational heterogeneity of cytochrome P450 3A4 revealed by high pressure spectroscopy. Biochem. Biophys. Res. Commun 2003;312:121130. [PubMed: 14630029]

17. Davydov DR, Botchkareva AE, Kumar S, He YQ, Halpert JR. An Electrostatically Driven Conformational Transition Is Involved in the Mechanisms of Substrate Binding and Cooperativity in Cytochrome P450eryF. Biochemistry 2004;43:6475-6485. [PubMed: 15157081]

18. Williams PA, Cosme J, Vinkovic DM, Ward A, Angove HC, Day PJ, Vonrhein C, Tickle IJ, Jhoti H. Crystal structures of human cytochrome P450 3A4 bound to metyrapone and progesterone. Science 2004;305:683-686. [PubMed: 15256616]

19. Roberts AG, Campbell AP, Atkins WM. The thermodynamic landscape of testosterone binding to cytochrome P450 3A4: ligand binding and spin state equilibria. Biochemistry 2005;44:1353-1366. [PubMed: 15667229]

20. Baas BJ, Denisov IG, Sligar SG. Homotropic cooperativity of monomeric cytochrome P450 3A4 in a nanoscale native bilayer environment. Arch. Biochem. Biophys 2004;430:218-228. [PubMed: 15369821] 
21. Dabrowski MJ, Schrag ML, Wienkers L, Atkins WM. Pyrene-pyrene complexes at the active site of cytochrome P450 3A4: Evidence for a multiple substrate binding site. J. Am. Chem. Soc 2002;124:11866-11867. [PubMed: 12358527]

22. Davydov DR, Kumar S, Halpert JR. Allosteric mechanisms in P450eryF probed with 1-pyrenebutanol, a novel fluorescent substrate. Biochem. Biophys. Res. Commun 2002;294:806-812. [PubMed: 12061778]

23. Reed JR, Hollenberg PF. Comparison of substrate metabolism by cytochromes P4502B1, 2B4, and 2B6: relationship of heme spin state, catalysis, and the effects of cytochrome $\mathrm{b}_{5}$. J. Inorg. Biochem 2003;93:152-160. [PubMed: 12576277]

24. Hlavica P, Lewis DFV. Allosteric phenomena in cytochrome P450-catalyzed monooxygenations. Eur. J. Biochem 2001;268:4817-4832. [PubMed: 11559350]

25. Blanck J, Ristau O, Zhukov AA, Archakov AI, Rein H, Ruckpaul K. Cytochrome P-450 spin state and leakiness of the monooxygenase pathway. Xenobiotica 1991;21:121-135. [PubMed: 1848383]

26. Rein H, Ristau O, Misselwitz R, Buder E, Ruckpaul K. The importance of the spin equilibrium in cytochrome P-450 for the reduction rate of the heme iron. Acta Biol. Med. Ger 1979;38:187-200. [PubMed: 229675]

27. Sligar SG, Cinti DL, Gibson GG, Schenkman JB. Spin state control of the hepatic cytochrome P450 redox potential. Biochem. Biophys. Res. Commun 1979;90:925-932. [PubMed: 228675]

28. Sligar SG. Coupling of spin, substrate, and redox equilibria in cytochrome P450. Biochemistry 1976;15:5399-5406. [PubMed: 187215]

29. Koley AP, Buters JT, Robinson RC, Markowitz A, Friedman FK. CO binding kinetics of human cytochrome P450 3A4. Specific interaction of substrates with kinetically distinguishable conformers. J Biol Chem 1995;270:5014-5018. [PubMed: 7890608]

30. Koley AP, Buters JTM, Robinson RC, Markowitz A, Friedman FK. Differential mechanisms of cytochrome P450 inhibition and activation by alpha-naphthoflavone. J. Biol. Chem 1997;272:31493152. [PubMed: 9013547]

31. Koley AP, Robinson RC, Markowitz A, Friedman FK. Drug-drug interactions: effect of quinidine on nifedipine binding to human cytochrome P450 3A4. Biochem. Pharm 1997;53:455-460. [PubMed: 9105395]

32. Davydov DR, Botchkareva AE, Davydova NE, Halpert JR. Resolution of two substrate-binding sites in an engineered cytochrome P450eryF bearing a fluorescent probe. Biophys. J 2005;89:418-432. [PubMed: 15834000]

33. Cupp-Vickery JR, Garcia C, Hofacre A, McGee-Estrada K. Ketoconazole-induced conformational changes in the active site of cytochrome P450eryF. J. Mol. Biol 2001;311:101-110. [PubMed: 11469860]

34. Khan KK, Halpert J. 7-benzyloxyquinoline oxidation by P450eryF A245T: Finding of a new fluorescent substrate probe. Chem. Res. Toxicol 2002;15:806-814. [PubMed: 12067248]

35. Job P. Recherches sur la formation de complexes minéraux en solution, et sur leur stabilityé. Ann. Chim. (Paris) 1928;9:113-203.

36. Ingham KC. Application of Job's method of continuous variation to stoichiometry of protein-ligand complexes. Anal. Biochem 1975;68:660-663. [PubMed: 1200365]

37. Facchiano A, Ragone R. Modification of Job's method for determining the stoichiometry of proteinprotein complexes. Anal. Biochem 2003;313:170-172. [PubMed: 12576074]

38. Domanski TL, He YA, Khan KK, Roussel F, Wang Q, Halpert JR. Phenylalanine and tryptophan scanning mutagenesis of CYP3A4 substrate recognition site residues and effect on substrate oxidation and cooperativity. Biochemistry 2001;40:10150-10160. [PubMed: 11513592]

39. Davydov DR, Deprez E, Hui Bon Hoa G, Knyushko TV, Kuznetsova GP, Koen YM, Archakov AI. High-Pressure-Induced Transitions in Microsomal Cytochrome P450 2B4 in Solution - Evidence for Conformational Inhomogeneity in the Oligomers. Arch. Biochem. Biophys 1995;320:330-344. [PubMed: 7625841]

40. Renaud JP, Davydov DR, Heirwegh KPM, Mansuy D, Hui Bon Hoa G. Thermodynamic studies of substrate binding and spin transitions in human cytochrome P-450 3A4 expressed in yeast microsomes. Biochem. J 1996;319:675-681. [PubMed: 8920966] 
41. Segel, IH. Enzyme Kinetics: Behavior and Analysis of Rapid Equilibrium and Steady-State Enzyme Systems. New York: Wiley-Interscience; 1975.

42. Cameron MD, Wen B, Allen KE, Roberts AG, Schuman JT, Campbell AP, Kunze KL, Nelson SD. Cooperative binding of midazolam with testosterone and alpha-naphthoflavone within the CYP3A4 active site: A NMR T-1 paramagnetic relaxation study. Biochemistry 2005;44:14143-14151. [PubMed: 16245930]

43. He YA, Roussel F, Halpert JR. Analysis of homotropic and heterotropic cooperativity of diazepam oxidation. Arch. Biochem. Biophys 2003;409:92-101. [PubMed: 12464248]

44. Shou, MG. Methods in Enzymology. vol. 357. New York: Academic Press; 2002. Kinetic analysis for multiple substrate interaction at the active site of cytochrome P450; p. 261-276.CYTOCHROME P450, PT C.

45. Galetin A, Clarke SE, Houston JB. Quinidine and haloperidol as modifiers of CYP3A4 activity: Multisite kinetic model approach. Drug Metab. Disp 2002;30:1512-1522.

46. Schrag ML, Wienkers LC. Covalent alteration of the CYP3A4 active site: Evidence for multiple substrate binding domains. Arch. Biochem. Biophys 2001;391:49-55. [PubMed: 11414684]

47. Hosea NA, Miller GP, Guengerich FP. Elucidation of distinct ligand binding sites for cytochrome P450 3A4. Biochemistry 2000;39:5929-5939. [PubMed: 10821664]

48. Davydov DR, Fernando H, Baas BJ, Sligar SG, Halpert JR. Kinetics of dithionite-dependent reduction of cytochrome P450 3A4: Heterogeneity of the enzyme caused by its oligomerization. Biochemistry 2005;44:13902-13913. [PubMed: 16229479] 

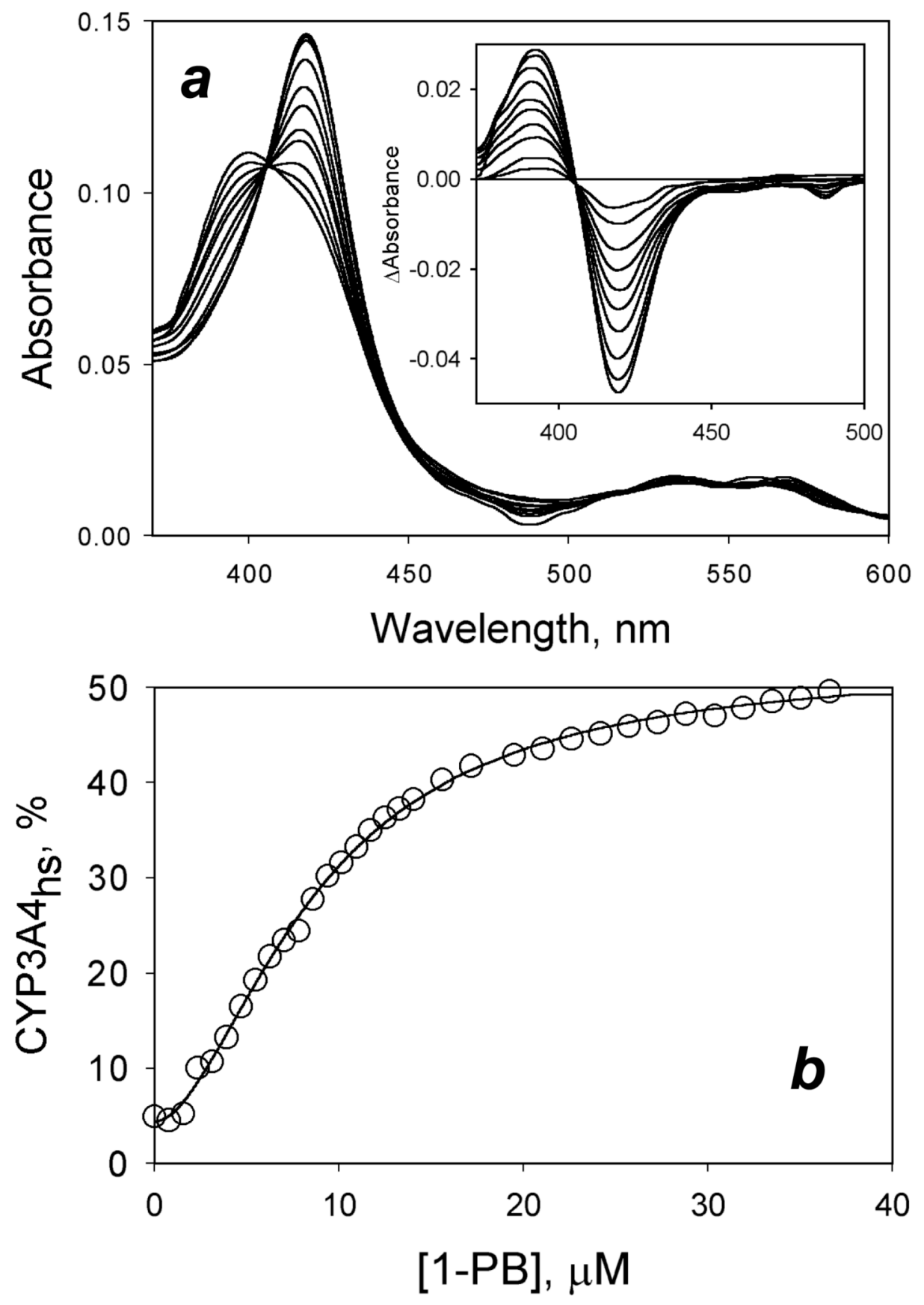

Figure 1.

Interactions of CYP3A4 with 1-PB monitored by the substrate-induced spin shift. (a) A series of the absorbance spectra obtained at no substrate present and at $0.78,1.6,3.1,5.5,7.8,10.1$, $11.7,17.2,25.7$, and 38.0 $\mu \mathrm{M}$ 1-PB. The insert shows the differential spectra obtained by subtraction of the first spectrum of the series (at no substrate present). (b) The same data shown as the plot of the percent of the high spin P450 versus the concentration of the substrate.. The line shows the approximation of this data set by the Hill equation with $S_{50}=8.6 \mu \mathrm{M}, n=1.8$, and the maximal amplitude of the spin shift of $48 \%$. The reaction mixture contained $1.45 \mu \mathrm{M}$ CYP3A4 in 0.1 M Na-Hepes buffer, pH 7.4, $1 \mathrm{mM}$ DTT, $1 \mathrm{mM}$ EDTA and was kept at $25^{\circ}$ C. 

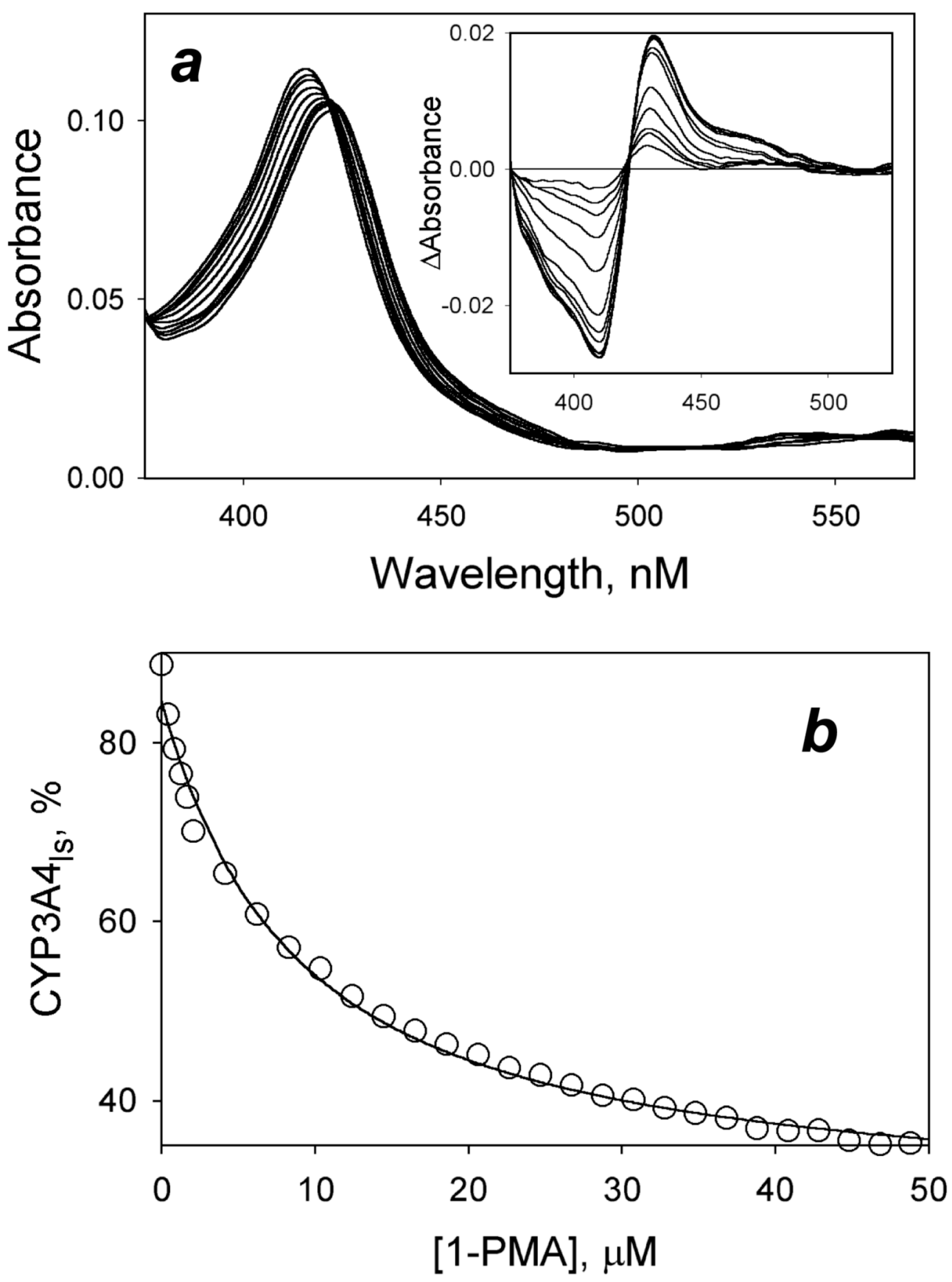

Figure 2.

Interactions of CYP3A4 with PMA monitored by the substrate-induced Type-II spectral transition. (a) A series of the absorbance spectra obtained at no substrate present and at 0.42 , 1.3, 1.7, 4.2 10.4, 12.4, 24.7, 30.8, and 48.9 $\mu$ M PMA. The insert shows the differential spectra obtained by subtraction of the first spectrum of the series (at no substrate present). (b) The same data shown as the fraction of the (water-ligated) low spin state P450 versus the concentration of the substrate. The solid line shows the approximation of this data set with a binary association equation with $K_{\mathrm{D}}=7.6 \mu \mathrm{M}$ and the maximal amplitude of the changes in the content of the P450 low-spin state of 58\%. The concentration of the enzyme was $1.2 \mu \mathrm{M}$. Other conditions as indicated in Fig. 1. 

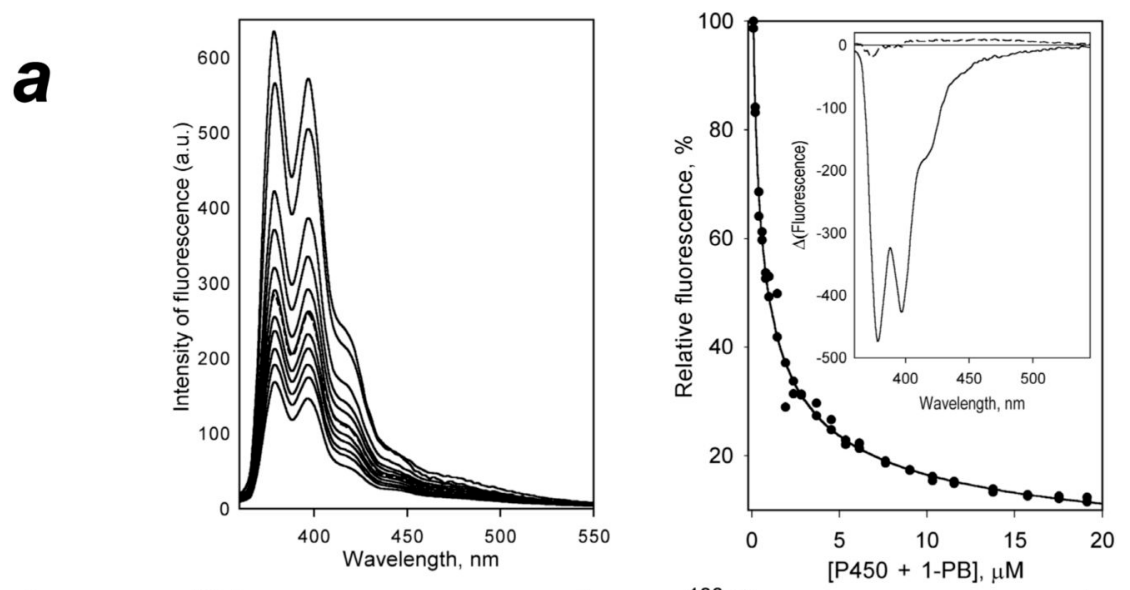

b
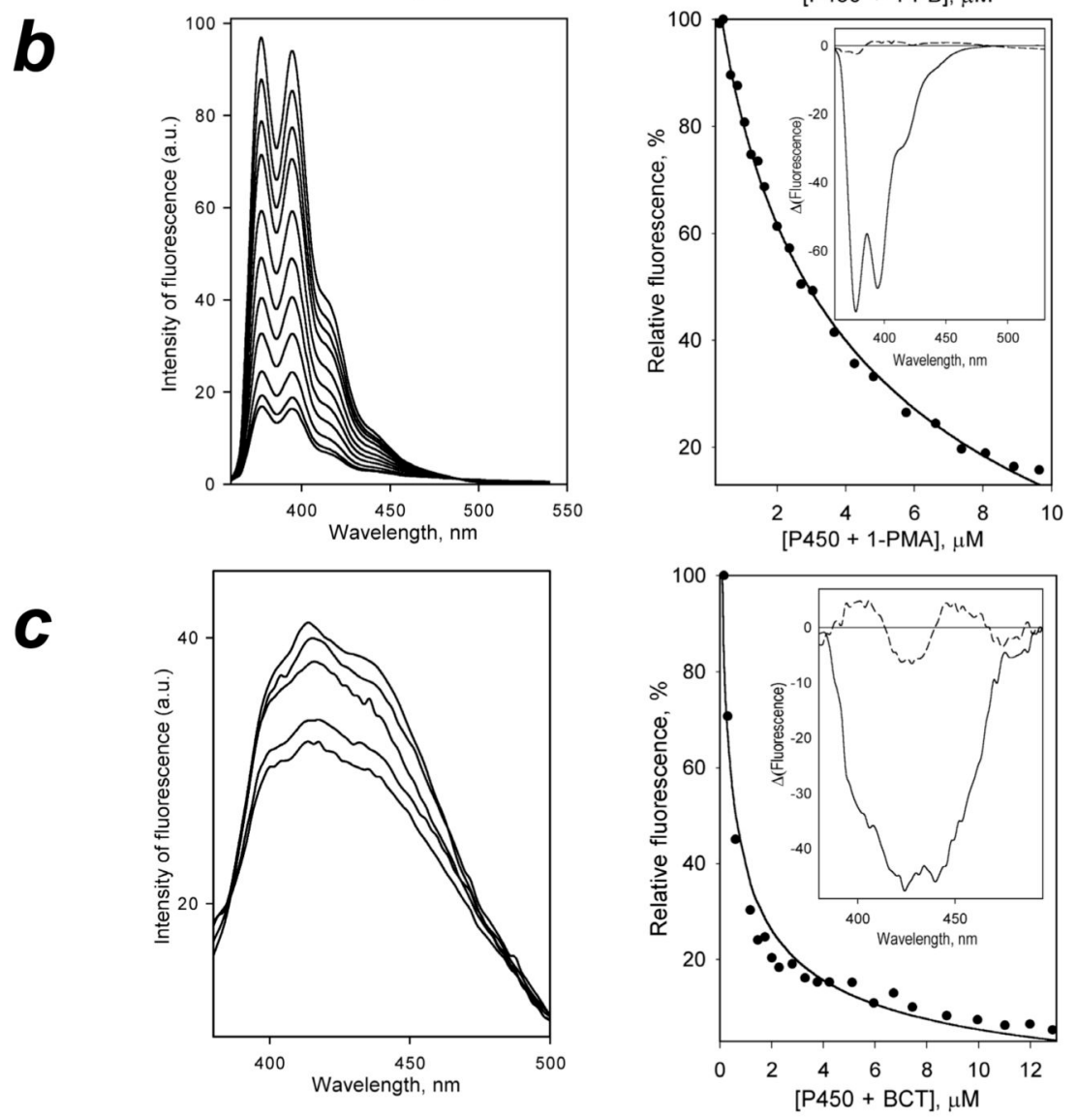

Figure 3.

Changes in specific fluorescence of 1-PB $(\boldsymbol{a})$, PMA $(\boldsymbol{b})$, and bromocriptine (c) upon dilution of the mixture of CYP3A4 with the respective substrates at a 1:1 molar ratio. The signal was normalized based on protein concentration and corrected for the internal filter effect as described. The left panels represent the series of fluorescence spectra measured at $0.26,0.53$, 1.0, 1.5, 2.0, 3.9, 4.7, 5.5, 6.3, 7.0, and $14.0 \mu \mathrm{M}$ (panel $\boldsymbol{a}$ ); 0.33, 0.64, 1.0, 1.4, 2.0, 2.7, 3.7, $4.8,6.6,8.1$, and $8.9 \mu \mathrm{M}$ (panel $\boldsymbol{b}$ ); and $1.9,1.6,1.4,1.1$ and $0.83 \mu \mathrm{M}$ enzyme (panel $\boldsymbol{c}$ ). In all cases the increase in the concentration of enzyme/substrate mixture caused a decrease in the normalized intensity of emission. The right panels represent the titration curves obtained from these data sets using principal component analysis, as described. The solid lines represent the 
approximations of the data sets by the equation for the equilibrium of bimolecular association with the values of $K_{\mathrm{D} 1}$ of $0.25 \mu \mathrm{M}, 3.1 \mu \mathrm{M}$, and $0.20 \mu \mathrm{M}$ for 1-PB, PMA, and bromocriptine respectively. The insets show the spectra of the first (solid lines) and the second (dashed lines) principal components scaled according to the maximal changes in the respective component observed in these titrations. Conditions as indicated in Fig 1. 

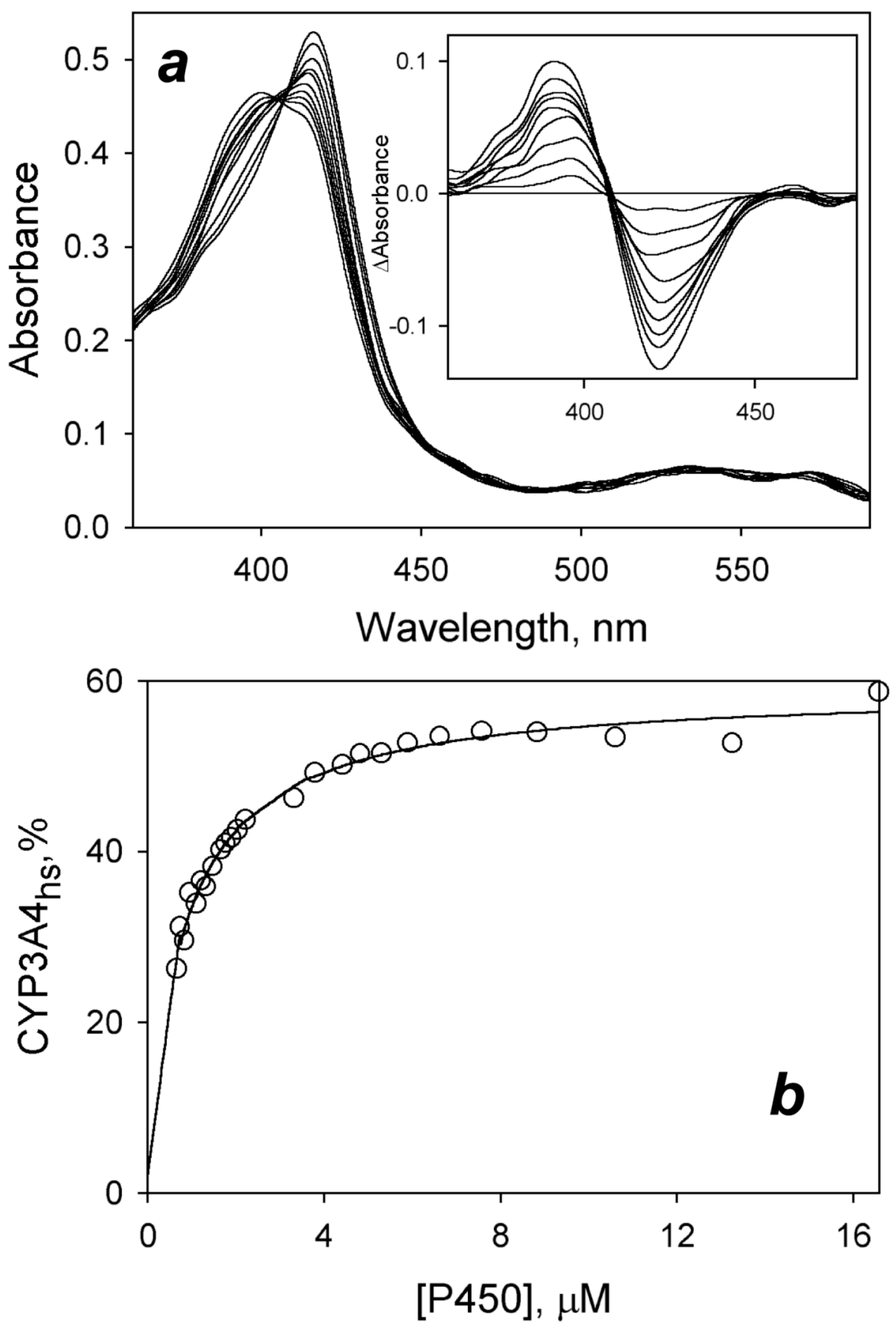

Figure 4.

Titration-by-dilution experiment on the interactions of CYP3A4 with 1-PB detected by absorbance spectroscopy at excess substrate. (a) A series of absorbance spectra of 1-PB in mixture with P450 3A4 at a 1:11 molar ratio. The spectra shown were obtained at 16.6 (initial concentration), 8.8, 5.3, 3.8, 3.3, 2.0, 1.5, 1.1, 0.83, and $0.66 \mu \mathrm{M}$ of CYP3A4. The inset shows a series of difference spectra of Fig. 4A. The experiment was carried out in a $10-\mathrm{cm}$ vertical cell with the optical pathlength increasing with the dilution of the sample. The amplitudes of the spectra were corrected for non-linearity of the dependence of the path length on the volume of the sample. (b) The spectral change shown in Fig. 4A as the plot of the fraction high spin P450 3A4 versus the concentration of the heme protein. The solid line shows the approximation 
of this data set with a binary association equation with a $K_{\mathrm{D} 2}=8.5 \mu \mathrm{M}$ and the maximal amplitude of the spin transition of 54\%. Conditions as indicated in Fig. 1. 

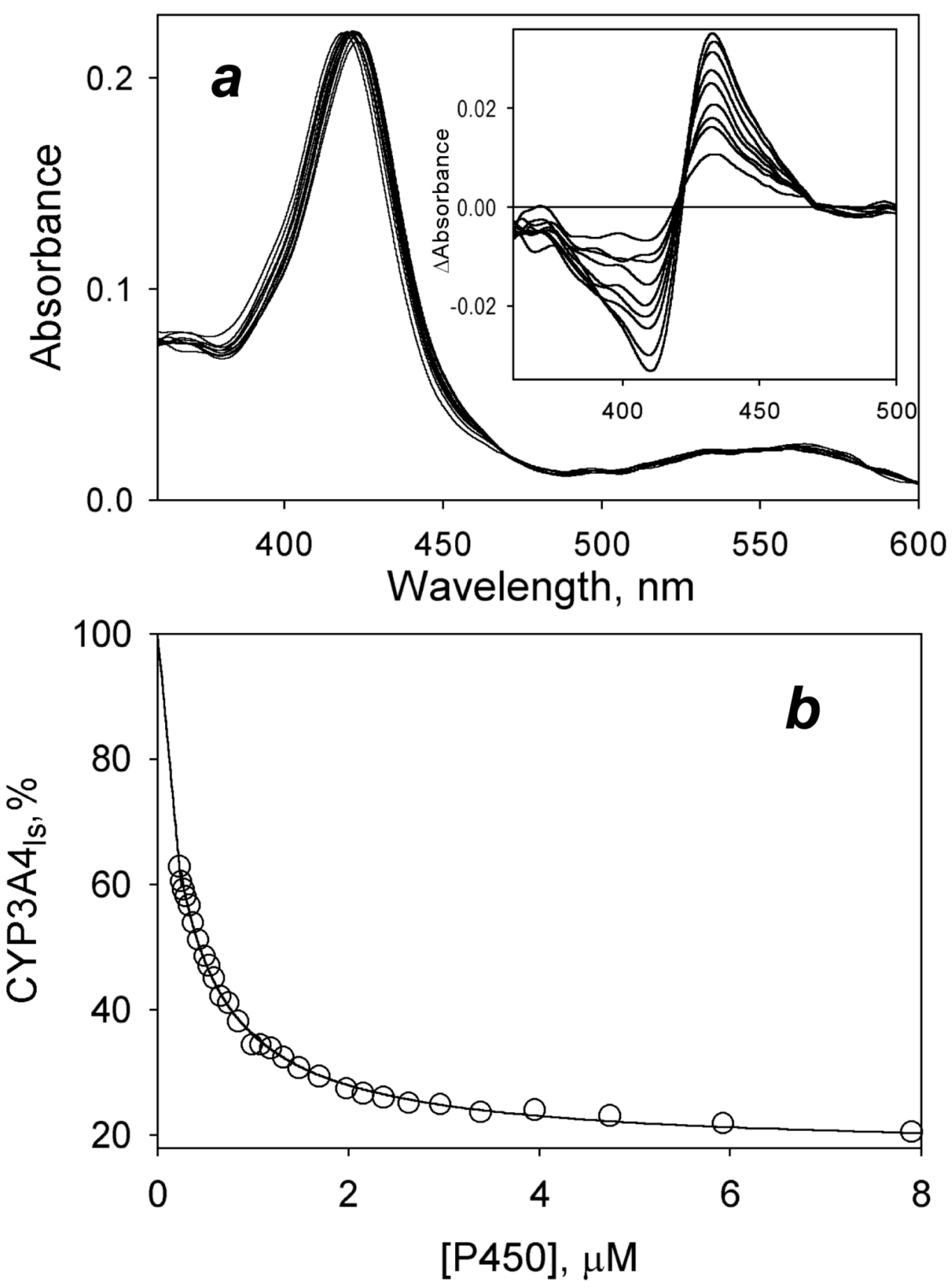

Figure 5.

Titration-by-dilution experiment on the interactions of CYP3A4 with PMA detected by absorbance spectroscopy at excess substrate. (a) A series of absorbance spectra of PMA in mixture with P450 3A4 at 1:13 molar ratio in a dilution setup. The spectra shown were obtained 32 at 7.9 (initial concentration), 4.0, 1.5, 1.1, 0.85, 0.66, 0.54, 0.49, 0.37, and $0.23 \mu \mathrm{M}$ of CYP3A4. The inset shows a series of difference spectra of Fig. 5A. The experiment was carried out in a 10-cm vertical cell with the optical pathlength increasing with the dilution of the sample. The amplitudes of the spectra were corrected for non-linearity of the dependence of the path length on the volume of the sample. (b) The spectral change shown in Fig. 5A as the plot of the fraction of low spin $\mathrm{P} 450$ versus the concentration of the heme protein. The solid line shows 
the approximation of this data set with a binary association equation with a $K_{\mathrm{D} 1}=3.6 \mu \mathrm{M}$ and the maximal amplitude of the changes in the content of the P450 low-spin state of $82 \%$. Conditions as indicated in Fig. 1. 

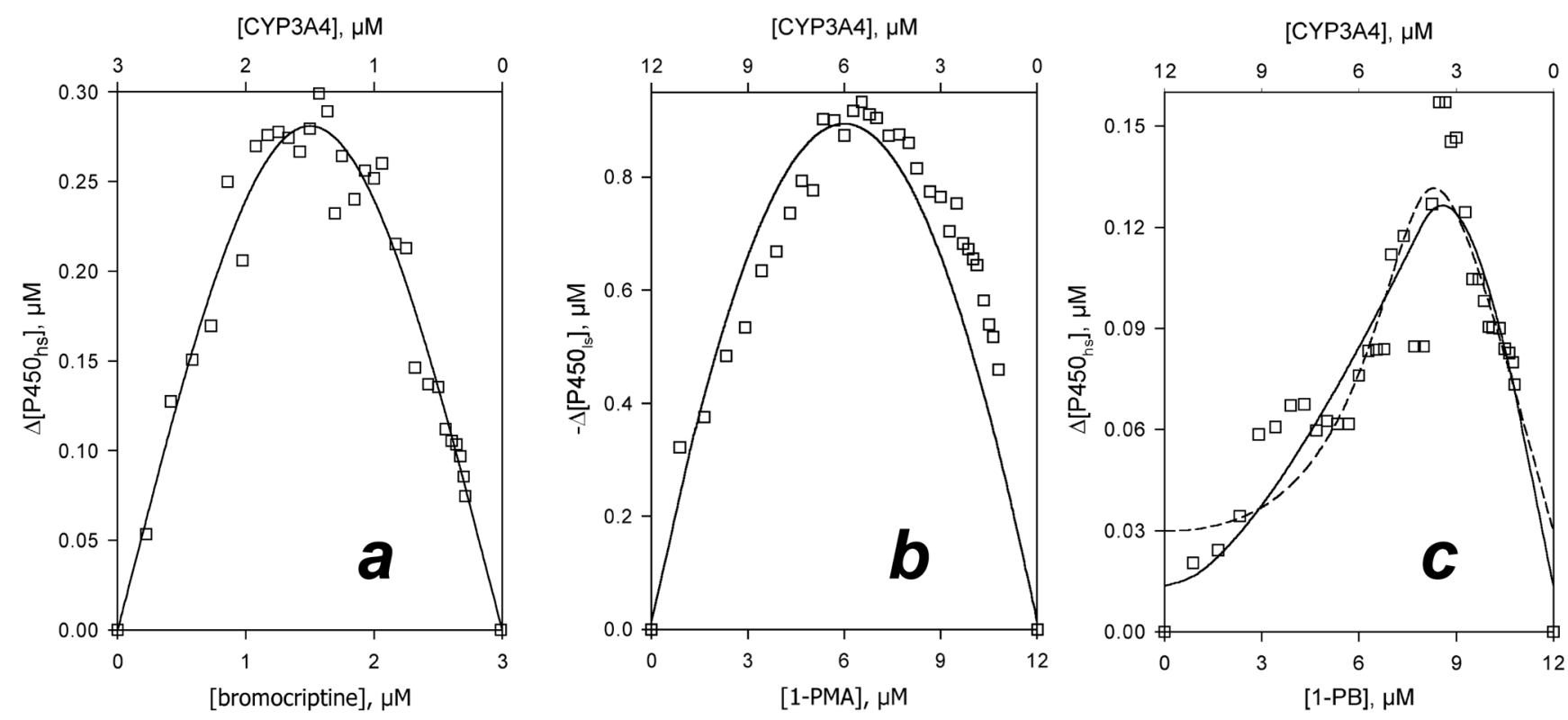

Figure 6.

Job's titration of CYP3A4 with bromocriptine (a), PMA (b), and 1-PB (c). The experiments were carried out at $25^{\circ} \mathrm{C}$ in a 10 -cm vertical cell with the optical pathlength increasing with the dilution of the sample. CYP3A4 $(500 \mu \mathrm{L})$ in $0.1 \mathrm{M} \mathrm{Na}$-Hepes buffer, pH 7.4, $1 \mathrm{mM}$ DTT, $1 \mathrm{mM}$ EDTA was placed into the optical cell, and the substrate solution of the same concentration as the initial protein was added gradually, until a volume of $5.1 \mathrm{~mL}$, when the cell was completely filled. The sum of the concentration of the enzyme and the substrate was therefore kept constant during the experiment, and was equal to $3 \mu \mathrm{M}$ in the titration with bromocriptine and $12 \mu \mathrm{M}$ in the experiments with 1-PB and PMA. The solid lines in panels (a) and $(\boldsymbol{b})$ represent the fitting of the experimental data with the bimolecular association isotherm (Eq. 1) with the values of dissociation constants of $0.38 \mu \mathrm{M}$ and $11.0 \mu \mathrm{M}$ for bromocriptine and PMA, respectively. The dashed line in panel (c) represents the approximation of the data with the parallel binding model (Eq. 3), while the solid line shows their approximation with the equation derived for the sequential binding mechanism (Eq. 4), assuming the value of $K_{\mathrm{D}} 1$ equal to $0.16 \mu \mathrm{M}$, as determined in our FRET exoperiments. The respective values of the dissociation constants of the ternary enzyme-substrate complex $\left(K_{D 2}\right)$ are equal to $0.55 \mu \mathrm{M}$ and $4.0 \mu \mathrm{M}$. 
Table 1

Parameters of the interactions of CYP3A4 with 1-PB, PMA, and bromocriptine.

\begin{tabular}{|c|c|c|c|c|}
\hline Method & \multirow{2}{*}{ Parameter } & \multicolumn{3}{|c|}{ Substrate } \\
\cline { 3 - 5 } & $S_{50}$ or $K_{D}, \mu \mathrm{M}$ & 1-PB & PMA & bromocriptine \\
\hline Spectrophotometric titration & $n$ & $8.0 \pm 1.1$ & $7.7 \pm 2.3$ & $0.31 \pm 0.08$ \\
& $A_{\max }, \% b$ & $1.8 \pm 0.3$ & N/A & N/A \\
& $K_{D}, \mu \mathrm{M}$ & $0.16 \pm 0.09$ & $4.8 \pm 1.4$ & $0.18 \pm 0.09$ \\
& $A_{\max }, \%^{c}$ & $92 \pm 19.2$ & $93 \pm 15$ & $96 \pm 8$ \\
\hline FRET Dilution experiments at 1:1[E]:[S] ratio & $K_{D}, \mu \mathrm{M}$ & $6.2 \pm 1.7$ & $5.8 \pm 1.8$ & N/D \\
\hline Titration-by-dilution at excess substrate & $K_{D}, \mu \mathrm{M}$ & $4.0 \pm 0.6$ & $11.0 \pm 0.7$ & $0.38 \pm 0.03$ \\
\hline Job's titration & &
\end{tabular}

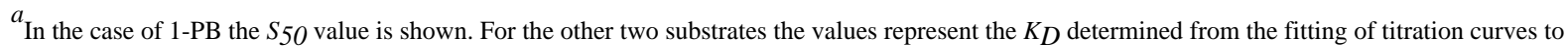
the equation (1).

${ }^{b}$ Maximal amplitude of substrate-induced changes in the content of the (water-ligated) low-spin CYP3A4. The values were deduced from the fitting of titration curves to an appropriate equation.

${ }^{c}$ The amplitude (efficiency) of FRET in terms of the relative decrease in the fluorescence of 1-PB upon its binding to CYP3A4. The values were determined from the fitting of titration curves to equation (1).

$d_{\text {De }}$

Determined from the approximation of the experimental data by the equation for the sequential binding model with two binding-sites. 